\title{
Temperature-independent strain sensor based on a tapered Bragg fibre fabricated using a $\mathrm{CO}_{2}$ laser
}

\author{
T. J. M. Martins ${ }^{1}$, M. B. Marques ${ }^{1}$, P. Roy ${ }^{2}$, R. Jamier ${ }^{2}$, S. Février ${ }^{2}$, O. Frazão ${ }^{1}$ \\ ${ }^{1}$ INESC TEC- Instituto de Engenharia de Sistemas e Computadores - Tecnologia e Ciência and \\ Department of Physics and Astronomy, Faculty of Sciences, University of Porto, Rua do Campo \\ Alegre 687, 4169-007 Porto, Portugal.
}

${ }^{2}$ XLIM - Institut de recherche, 123, avenue Albert Thomas - 87060 LIMOGES CEDEX.

\begin{abstract}
Temperature-independent strain measurement is achieved resorting to a taper fabricated on a Bragg fibre using a $\mathrm{CO}_{2}$ laser. The characteristic bimodal interference of an untapered Bragg fibre is rendered multimode after taper fabrication and the resulting transmission spectra are analysed as temperature and strain change. The intrinsic strain sensitivity exhibited by the Bragg fibre is increased 15 fold after tapering and reaches $22.68 \mathrm{pm} / \mu \varepsilon$. The difference in wavelength shift promoted by variations in temperature and strain for the two fringes studied is examined and strain sensing with little temperature sensitivity is achieved, presenting a sensitivity of $2.86 \mathrm{pm} / \mu \varepsilon$, for strain values up to $400 \mu \varepsilon$.
\end{abstract}

Keywords: Bragg fibre, optical fibre sensor, taper, interferometer.

\section{INTRODUCTION}

Optical fibre technology has met many breakthroughs over the last decades, such as the development of more complex structures like Bragg fibres, whose theoretical model was proposed in $1978^{[1]}$. These fibres are cylindrical waveguides consisting of a low-index core surrounded by concentric rings of material with alternating high and low refractive index, acting as a cylindrical Bragg mirror. The range of applications spans a wide variety of possibilities, from high-power fibre lasers, since Bragg fibres allow the propagation of a single mode with a large effective area ${ }^{[2]}$, to supercontinuum generation ${ }^{[3]}$ and dispersion compensation ${ }^{[4]}$, making the best use of the fact that the dispersion characteristics of Bragg fibres can be controlled by tailoring the periodic index modulation of the cladding. Optical sensing has also benefited from the introduction of Bragg fibres, allowing strain and temperature discrimination ${ }^{[5]}$. The underlying problem of cross-sensitivity that motivates the creative effort of designing devices capable of physical parameter discrimination has resulted in several other proposals ${ }^{[6,7]}$.

This paper presents the evaluation of the behaviour of a taper fabricated on a Bragg fibre, using a $\mathrm{CO}_{2}$ laser, as a sensor of strain and temperature. In the 1550-nm region, the bimodal interference between the fundamental mode and a high order mode exhibited by the untapered fibre is transformed in a multimode interference after the taper fabrication. The interference conditions created within the tapered Bragg fibre are monitored as both strain and temperature change by interrogating the sensor in transmission.

Sixth European Workshop on Optical Fibre Sensors, edited by Elfed Lewis, Proc. of SPIE Vol. 9916, 991610 - (c) 2016 SPIE · CCC code: 0277-786X/16/\$18 · doi: 10.1117/12.2235239 


\section{EXPERIMENTAL RESULTS}

The proposed interferometric sensor is based on a taper fabricated on a stretch of hexagonal Bragg fibre with $5 \mathrm{~cm}$ resorting to a SYNRAD 48-1 $\mathrm{CO}_{2}$ laser providing IR radiation with $10.6 \mu \mathrm{m}$ wavelength and $10 \mathrm{~W}$ maximum output power. The $5 \mathrm{~cm}$ Bragg fibre is spliced at both ends with a standard singlemode fibre (SMF) and fixed onto AEROTECH motorized stages controlled via PC. These two stages move in the same direction with different speeds, set equal to $135 \mu \mathrm{m} / \mathrm{s}$ and $40 \mu \mathrm{m} / \mathrm{s}$, placing the fibre under additional mechanical stress. Laser power is $1.8 \mathrm{~W}$ and the taper length is chosen to be $5 \mathrm{~mm}$. The fabrication process consists of a double sweep of the optical fibre by the $\mathrm{CO}_{2}$ laser beam, yielding a $5 \mathrm{~mm}$ thin taper with a $35 \mu \mathrm{m}$ waist diameter. As observed through Figure 1 (a), the fibre profile is kept almost unchanged after taper fabrication, except when it comes to its characteristic dimensions, considerably reduced: the three rings as well as the hexagonal border, considered just as an outer ring (circular profile), experience diameter reductions with ratios between 1:3.3 and 1:3.6. The tapered Bragg fibre is interrogated in transmission using a broadband light source with a $1550 \mathrm{~nm}$ central wavelength and a $100 \mathrm{~nm}$ bandwidth as well as an ADVANTEST Q8384 Optical Spectrum Analyzer (OSA). The transmission spectra are studied setting the OSA resolution to $0.02 \mathrm{~nm}$.

The intrinsic structure of the untapered Bragg fibre, consisting of a low-index core and a circularly periodic index modulation of the cladding, creates a bimodal interference. After taper fabrication, the interference becomes multimode as more high order modes are excited as seen in Figure 1 (b). The average transmitted power is significantly decreased meaning that indeed light is being coupled from the fundamental mode, mainly guided through the core, to higher order modes in the cladding.

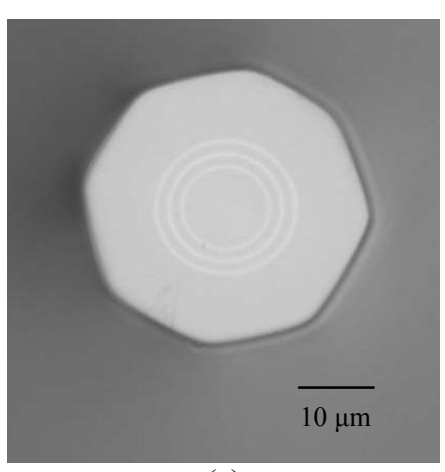

(a)

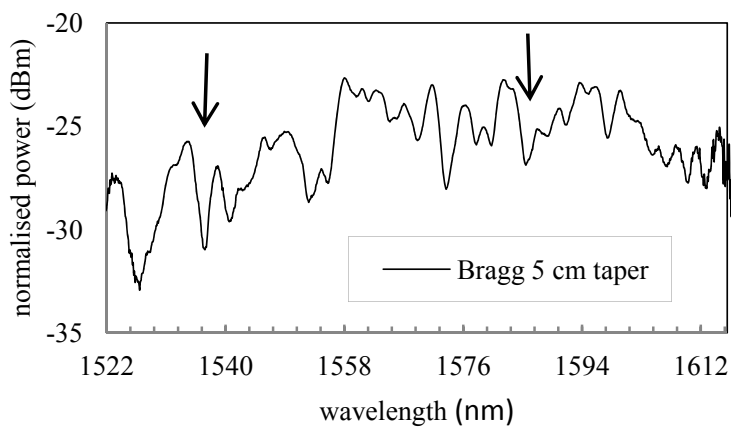

(b)

Figure 1 - Top view of the tapered Bragg fibre (a) and transmission spectra for a $5 \mathrm{~cm}$ long stretch of tapered Bragg fibre (b). The arrows identify the fringes monitored during strain characterisation (for zero strain applied).

The experimental setup designed for strain characterisation is quite straightforward and besides the broadband light source and the spectrum analyser it only includes a manually controlled translation stage in order to change the tension applied to the fibre by varying the distance between the two points where the fibre is fixed: on top of the very same translation stage and on a magnetised block placed at a certain distance.

The monitoring procedure is carried out by determining the location of two fringes in the interference spectrum of the tapered Bragg fibre, located at $\lambda_{1}=1536 \mathrm{~nm}$ and $\lambda_{2}=1584 \mathrm{~nm}$, as the applied strain increases. In order to determine whether the tapering process promotes a significant increase in the sensitivity of the device, the $5 \mathrm{~cm}$ long unchanged Bragg fibre is also monitored. The fringes considered are located at $\lambda_{1}=1547 \mathrm{~nm}$ and $\lambda_{2}=1582 \mathrm{~nm}$. The deviations concerning fringe position between both the tapered and untaperd fibres are under $1 \%$, which is important if one wants a reasonable and conclusive sensitivity comparison. The graphic in Figure 2 clearly shows an increase in 
sensitivity as a result of the tapering process for both fringes. The calculated sensitivities are, from the shortest to the longest fringe wavelength, $22.68 \mathrm{pm} / \mu \varepsilon$ and $20.02 \mathrm{pm} / \mu \varepsilon$, for the tapered fibre, and $1.51 \mathrm{pm} / \mu \varepsilon$ and $1.78 \mathrm{pm} / \mu \varepsilon$, for the untapered fibre. The maximum increase is then of approximately 15 times and is registered for the fringe at shorter wavelengths. The calculated values for these strain sensitivities surpass those presented in some of the literature ${ }^{[6,7]}$, though verified over a smaller range of applied strain. Temperature characterisation of the tapered Bragg fibre follows a similar set of steps to the one employed to perform strain characterisation. The taper is placed inside an oven to allow raising its temperature up to $450^{\circ} \mathrm{C}$. The examined fringes are located at $\lambda_{1}=1535 \mathrm{~nm}$ and $\lambda_{2}=1572 \mathrm{~nm}$ and the promoted wavelength shifts due to changing temperature are registered and displayed in Figure 3 . Regarding fringe location, the maximum deviation from the values considered for strain characterisation is under $1 \%$. The sensitivities are, from the shortest to the longest fringe wavelength, $-12.11 \mathrm{pm} /{ }^{\circ} \mathrm{C}$ and $-12.20 \mathrm{pm} /{ }^{\circ} \mathrm{C}$, also larger than some reported results ${ }^{[6,7]}$.

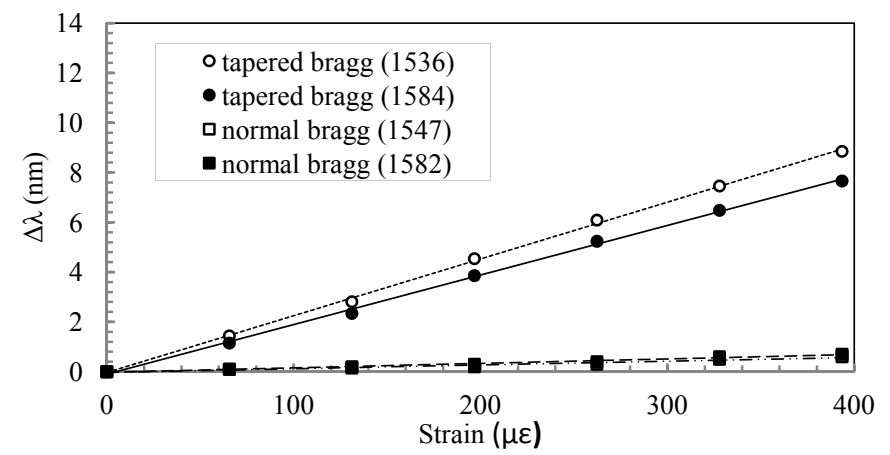

Figure $2-$ Wavelength shift $\Delta \lambda$ as a function of the applied strain for both the tapered and the untapered Bragg fibres. Linear fits for all the considered fringes are displayed

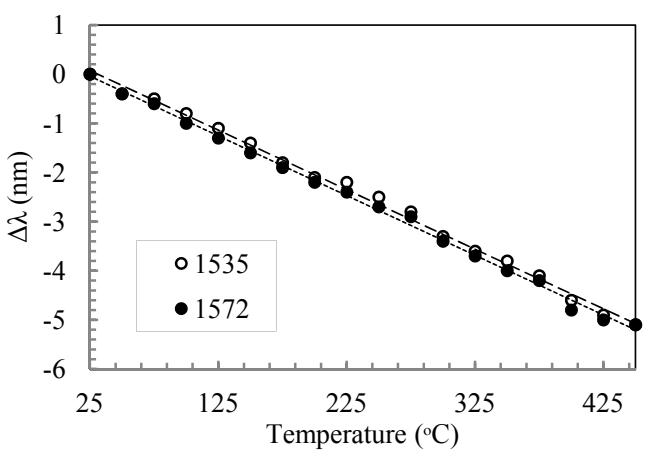

Figure 3 - Wavelength shift $\Delta \lambda$ as a function of the temperature for the tapered Bragg fibre. Linear fits for both the considered fringes are displayed.

The difference between fringe sensitivities is larger in the case of applied strain. The study of the difference in fringe location $\lambda_{2}-\lambda_{1}$ makes it apparent the possibility of strain sensing with little temperature sensitivity as observed through Figure 4 . These experimental results agree well with our early expectations: only the perturbations that change the geometry of this device, such as strain, are expected to give rise to different sensitivities for different interference fringes. In fact, the new sensitivities, obtained resorting to this sensing scheme, equal to $2.86 \mathrm{pm} / \mu \varepsilon$ and $-0.04 \mathrm{pm} / \mathrm{K}$, show that strain monitoring can be performed with a sensitivity approximately 70 times larger than the one associated to temperature.

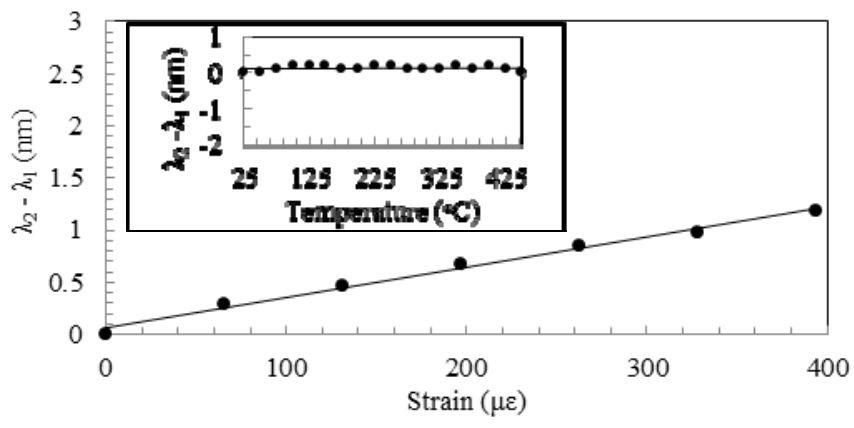

Figure 4 - Wavelength shift $\Delta \lambda$ difference as a function of the applied strain for the tapered Bragg fibre. Inset: wavelength shift $\Delta \lambda$ difference as a function of the temperature for the tapered Bragg fibre. Linear fits for both graphics. 


\section{CONCLUSION}

The $\mathrm{CO}_{2}$-supported tapering process changes the spatial profile of a Bragg fibre altering the interference created inside the fibre, moving from a bimodal to a multimode interference as light couples from the core to excite higher order cladding modes. The resulting changes in strain sensitivity are remarkable with values reaching $22.68 \mathrm{pm} / \mu \varepsilon$, which represents an increase of 15 times the sensitivity of an untapered Bragg fibre. The obtained temperature sensitivities, reaching $-12.20 \mathrm{pm} / \mathrm{K}$, are also worth mentioning, along with the fact that the range of temperature values covered goes from $25^{\circ} \mathrm{C}$ up to $450{ }^{\circ} \mathrm{C}$. A temperature-independent strain sensor based on the same tapered Bragg fibre is also proposed with a sensitivity of $2.86 \mathrm{pm} / \mu \varepsilon$, still larger than some of the reported values in the literature, and with a strain variation due to changes in temperature equal to $0.01 \mu \varepsilon / \mathrm{K}$.

\section{ACKNOWLEDGEMENTS}

The authors acknowledge S. L. Semjonov, M. E. Likhachev, M. M. Bubnov, and E. M. Dianov from the Fiber Optics Research Center, Moscow, and V. F. Khopin, M. Y. Salganskii, and A. N. Guryanov from the Institute of Chemistry of High Purity Substances, and N. Novgorod for the fabrication of the fiber samples.

This work is financed by the ERDF - European Regional Development Fund through the Operational Programme for Competitiveness and Internationalisation - COMPETE 2020 Programme, and by National Funds through the FCT Fundação para a Ciência e Tecnologia (Portuguese Foundation for Science and Technology) within project POCI-010145-FEDER-006961.

\section{REFERENCES}

[1] Yeh, P., Yariv, A., Marom, E., “Theory of Bragg fiber”, J. Opt. Soc. Am., 68(8), 1196-1201 (1978).

[2] Février, S., Viale, P., Gérôme, F., Leproux, P., Roy, P., Blondy, J.-M., Dussardier, B., Monnom, G., "Very large effective area singlemode photonic bandgap fibre", Electron. Lett., 39(2), 140-1242 (2003).

[3] Jamier R., Ducros, N., Février, S., Likhachev, M. E., Salganskii, M. Y., "Tight control of the spectral broadening obtained by nonlinear conversion in a photonic bandgap fiber", Proc. CLEO/IQEC (2009), Paper JWA53.

[4] Gérôme, F., Février, S., Pryamikov, A. D., Auguste, J.- L., Jamier, R., Blondy, J.-M., Likhachev, M. E., Bubnov, M. M., Semjonov, S. L., Dianov, E. M., "Highly dispersive large mode area photonic bandgap fiber", Opt. Lett. 32(10), 1208-1210 (2007).

[5] Frazão, O., Amaral, L. M. N., Baptista, J. M., Roy, P., Jamier, R., Février, S., "Strain and temperature discrimination using modal interferometry in Bragg fibers, IEEE Photon. Tech. Lett., 22(21), 1616-1618 (2010).

[6] Frazão, O., Melo, M. A., Marques, P. V. S., Santos, J. L., "Chirped Bragg grating fabricated in fused fibre taper for strain-temperature discrimination", Meas. Sci. Technol. 16(4), 984-988 (2005).

[7] Frazão, O., Marques, L., Marques, J. M., Baptista, J. M., Santos, J. L., "Simple sensing head geometry using fibre Bragg gratings for strain-temperature discrimination", Opt. Comm., 279(1), 68-71 (2007). 